\title{
FIRST PASSAGE PERCOLATION HAS SUBLINEAR DISTANCE VARIANCE
}

\author{
By ItAi BenJamini, Gil Kalai ANd Oded SCHRAMm
}

\section{Weizmann Institute of Science, Hebrew University and Microsoft Research}

Let $0<a<b<\infty$, and for each edge $e$ of $\mathbb{Z}^{d}$ let $\omega_{e}=a$ or $\omega_{e}=b$, each with probability $1 / 2$, independently. This induces a random metric dist $\omega$ on the vertices of $\mathbb{Z}^{d}$, called first passage percolation. We prove that for $d>1$, the distance $\operatorname{dist}_{\omega}(0, v)$ from the origin to a vertex $v,|v|>2$, has variance bounded by $C|v| / \log |v|$, where $C=C(a, b, d)$ is a constant which may only depend on $a, b$ and $d$. Some related variants are also discussed.

1. Introduction. Consider the following model of first passage percolation. Fix some $d=2,3, \ldots$ and let $E=E\left(\mathbb{Z}^{d}\right)$ denote the set of edges in $\mathbb{Z}^{d}$. Also fix numbers $0<a<b<\infty$. Let $\Omega:=\{a, b\}^{E}$ carry the product measure, where $\mathbf{P}\left[\omega_{e}=a\right]=\mathbf{P}\left[\omega_{e}=b\right]=1 / 2$ for each $e \in E$. Given $\omega=\left(\omega_{e}: e \in E\right) \in \Omega$ and vertices $v, u \in \mathbb{Z}^{d}$, let $\operatorname{dist}_{\omega}(v, u)$ denote the least distance from $v$ to $u$ in the metric induced by $\omega$; that is, the infimum of $\sum_{e \in \alpha} \omega_{e}$, where $\alpha$ ranges over all finite paths in $\mathbb{Z}^{d}$ from $u$ to $v$. Let $|v|:=\|v\|_{1}$ for vertices $v \in \mathbb{Z}^{d}$.

THEOREM 1. There is a constant $C=C(d, a, b)$ such that for every $v \in \mathbb{Z}^{d}$, $|v| \geq 2$,

$$
\operatorname{var}\left(\operatorname{dist}_{\omega}(0, v)\right) \leq C \frac{|v|}{\log |v|}
$$

Kesten [12] used martingale inequalities to prove $\operatorname{var}\left(\operatorname{dist}_{\omega}(0, v)\right) \leq C|v|$ and proved some tail estimates. Talagrand [17] used his "convexified" discrete isoperimetric inequality to prove that for all $t>0$,

$$
\mathbf{P}\left[\left|\operatorname{dist}_{\omega}(0, v)-M\right| \geq t \sqrt{|v|}\right] \leq C \exp \left(-t^{2} / C\right),
$$

where $M$ is the median value of $\operatorname{dist}_{\omega}(0, v)$. (Both Kesten's and Talagrand's results apply to more general distributions of the edge lengths $\omega_{e}$.) "Novice readers might expect to hear next of a central limit theorem being proved," writes Durrett [7], describing Kesten's results, "however physicists tell us... that in two dimensions the standard deviation... is of order $|v|^{1 / 3}$." Recent remarkable work $[1,6,9]$ not only supports this prediction, but suggests what the limiting distribution and large deviation behavior is. The case of a certain variant of oriented first passage

Received April 2002; revised October 2002.

AMS 2000 subject classifications. Primary 60K35; secondary 60B15, 28A35, 60E15.

Key words and phrases. Hypercontractive, harmonic analysis, discrete harmonic analysis, discrete cube, random metrics, discrete isoperimetric inequalities, influences.

1970

I. Benjamini, O. Häggström (eds.), Selected Works of Oded Schramm, Selected Works in Probability and Statistics, DOI 10.1007/978-1-4419-9675-6_26, C Springer Science+Business Media, LLC 2011 
percolation is settled by Johansson [9]. For lower bounds on the variance in twodimensional first passage percolation, see [14, 15].

As in Kesten's and Talagrand's earlier results, the most essential feature about first passage percolation which we use is that the number of edges $e \in E$ such that modifying $\omega_{e}$ increases $\operatorname{dist}_{\omega}(0, v)$ is bounded by $C|v|$.

Another essential ingredient in the current article is the following extension by Talagrand [16] of an inequality by Kahn, Kalai and Linial [10]. Let $J$ be a finite index set. For $j \in J$ and $\omega \in\{a, b\}^{J}$, let $\sigma_{j} \omega$ be the element of $\{a, b\}^{J}$ which is different from $\omega$ only in the $j$ th coordinate. For $f:\{a, b\}^{J} \rightarrow \mathbb{R}$ set $\sigma_{j} f:=f \circ \sigma_{j}$ and

$$
\rho_{j} f(\omega):=\frac{f(\omega)-\sigma_{j} f(\omega)}{2} .
$$

Talagrand's ([16], Theorem 1.5) inequality is

$$
\operatorname{var}(f) \leq C \sum_{j \in J} \frac{\left\|\rho_{j} f\right\|_{2}^{2}}{1+\log \left(\left\|\rho_{j} f\right\|_{2} /\left\|\rho_{j} f\right\|_{1}\right)},
$$

where $C$ is a universal constant. [In Section 4 we supply a direct proof of (2) from the Bonami-Beckner inequality. A very reasonable upper bound for $C$ can be obtained from this proof. We also explain there how (2) easily implies (1).]

The basic idea in the proof of Theorem 1 is to apply this inequality to $f(\omega):=$ $\operatorname{dist}_{\omega}(0, v)$. For this, we wanted to show that, roughly, $\mathbf{P}\left[\rho_{e} f(\omega) \neq 0\right]$ is small, except for a small number of edges $e$. However, since we were not able to prove this, we had to resort to an averaging trick.

Theorem 1 should hold for other models of first passage percolation, where the edge lengths have more general distributions. The relevant result of [10], as well as (2) that we use rely on the Bonami-Beckner [2, 4] inequality, which holds for $\{a, b\}^{n}$, but fails on some more general product spaces. Bourgain, Kahn, Kalai, Katznelson and Linial [5] extended some of these results to general product spaces; see also [8]. Talagrand's ([16], Theorem 1.5) result applies to product measures on $\{a, b\}^{n}$, which are not necessarily uniform. Beckner [2] used his inequality to derive a similar result for the Gaussian measure on $\mathbb{R}^{n}$, and an analog for Talagrand's inequality (2) for the Gaussian measure (pointed out to us by Assaf Naor) can be found in [3]. In the present article, we prefer simplicity to generality, but it will be interesting to extend the theorem to more general distributions. We refer the reader also to the book by Ledoux [13] for a general view of relevant techniques and knowledge.

It seems even more important to put effort into the fundamental task of lowering the upper bound from $C|v| / \log |v|$ to $C|v|^{1-\varepsilon}, \varepsilon>0$.

To first illustrate the basic technique in a simpler setting, we also prove the following theorem about the variance of the first passage percolation diameter in 
graphs with symmetries. If $G=(V(G), E(G))$ is a finite connected graph and $\omega: E(G) \rightarrow\{a, b\}$, define

$$
\operatorname{diam}_{\omega}(G):=\max _{v, u \in V(G)} \operatorname{dist}_{\omega}(v, u) .
$$

(Although we have defined dist $\omega$ only for $\mathbb{Z}^{d}$, the definition obviously extends to arbitrary graphs.) Also, let $\operatorname{diam}(G)$ denote the diameter of $G$ in the graph metric; that is, $\operatorname{diam}(G)=\operatorname{diam}_{1}(G)$.

THEOREM 2. Let $G=(V(G), E(G))$ be a finite connected graph and let $\Gamma$ be the group of automorphisms of $G$. Set $Y:=\min \{|\Gamma e|: e \in E(G)\}$; that is, the cardinality of the smallest orbit of edges under $\Gamma$. Let $\omega \in\{a, b\}^{E(G)}$ be random uniform. Then

$$
\operatorname{var}\left(\operatorname{diam}_{\omega} G\right) \leq C \frac{(b-a)^{2}(b / a) \operatorname{diam} G}{1+\log \max \{1,(a / b) Y / \operatorname{diam} G\}},
$$

where $C$ is a universal constant.

Also consider first passage percolation on an $m \times n$ discrete torus $(\mathbb{Z} / m \mathbb{Z}) \times$ $(\mathbb{Z} / n \mathbb{Z})$. Among all simple closed paths whose projection onto the first coordinate has degree 1 (i.e., every edge in $\mathbb{Z} / m \mathbb{Z}$ is traversed by the projected walk one time more in one direction than in the other), there is at least one which has minimal $\omega$-length. Call this length the circumference of $\omega$. One can apply the proof of Theorem 2 to show that the variance of the circumference is $o(n)$ when $m, n \rightarrow \infty$.

2. Proof of Theorem 2. Let $f(\omega):=\operatorname{diam}_{\omega}(G)$. Let $u_{1}, u_{2} \in V(G)$ be a pair of vertices where $\operatorname{dist}_{\omega}\left(u_{1}, u_{2}\right)=\operatorname{diam}_{\omega}(G)$ and let $\beta$ be a path from $u_{1}$ to $u_{2}$ such that $\sum_{e \in \beta} \omega_{e}=\operatorname{diam}_{\omega}(G)$. We use some arbitrary but fixed method for choosing between all possible choices for the triple $\left(u_{1}, u_{2}, \beta\right)$. Clearly, $\operatorname{diam}_{\omega}(G) \leq b \cdot \operatorname{diam}(G)$. Therefore,

$$
|\beta| \leq(b / a) \operatorname{diam}(G),
$$

where $|\beta|$ denotes the number of edges in $\beta$.

Let $e \in E(G)$. Note that if $\rho_{e} f(\omega)<0$, then we must have $e \in \beta$. By (3), this gives

$$
\sum_{e \in E(G)} \mathbf{P}\left[\rho_{e} f(\omega)<0\right] \leq \mathbf{E}[|\beta|] \leq(b / a) \operatorname{diam}(G) .
$$

Fix $e \in E(G)$. By symmetry, $\mathbf{P}\left[\rho_{e} f<0\right]=\mathbf{P}\left[\rho_{e^{\prime}} f<0\right]$ for every $e^{\prime} \in \Gamma e$. Consequently,

$$
|\Gamma e| \mathbf{P}\left[\rho_{e} f<0\right] \leq \sum_{e^{\prime} \in E(G)} \mathbf{P}\left[\rho_{e^{\prime}} f<0\right] \leq(b / a) \operatorname{diam}(G) .
$$


Now clearly, $\mathbf{P}\left[\rho_{e} f \neq 0\right]=2 \mathbf{P}\left[\rho_{e} f<0\right]$ and $\left\|\rho_{e} f\right\|_{\infty} \leq(b-a) / 2$. Therefore,

$$
\left\|\rho_{e} f\right\|_{2}^{2} \leq(1 / 2)(b-a)^{2} \mathbf{P}\left[\rho_{e} f<0\right] .
$$

By Cauchy-Schwarz,

$$
\left\|\rho_{e} f\right\|_{1} \leq \sqrt{\mathbf{P}\left[\rho_{e} f \neq 0\right]}\left\|\rho_{e} f\right\|_{2} .
$$

By (2), we have

$$
\operatorname{var}(f) \leq C \frac{\sum_{e \in E(G)}\left\|\rho_{e} f\right\|_{2}^{2}}{1+\min _{e \in E(G)} \log \left(\left\|\rho_{e} f\right\|_{2} /\left\|\rho_{e} f\right\|_{1}\right)} .
$$

To estimate the numerator, we use (6) and (4), and for the denominator, (5) and (7). The theorem easily follows.

3. Proof of Theorem 1. If $v, u \in \mathbb{Z}^{d}$, and $\alpha$ is a path from $v$ to $u$, then $\alpha$ is called an $\omega$-geodesic if it minimizes $\omega$-length; that is, dist $\omega=\sum_{e \in \alpha} \omega_{e}$. Given $\omega$, let $\gamma$ be an $\omega$-geodesic from 0 to $v$. Although there may be more than one such geodesic, we require that $\gamma$ depends only on $\omega$. (E.g., we may use an arbitrary deterministic choice among any possible collection of $\omega$-geodesics.)

The general strategy for the proof of Theorem 1 is as for Theorem 2. However, the difficulty is that there is not enough symmetry to get a good bound on $\mathbf{P}\left[\rho_{e} \operatorname{dist}_{\omega}(0, v)<0\right]$. It would have been enough to show that $\mathbf{P}[e \in \gamma]<$ $C|v|^{-1 / C}$ holds with the exception of at most $C|v| / \log |v|$ edges, for some constant $C>0$, but we could not prove this. Therefore, we need an averaging argument, for which the following lemma is useful.

LEMMA 3. There is a constant $c>0$ such that for every $m \in \mathbb{N}$ there is a function

$$
g=g_{m}:\{a, b\}^{m^{2}} \rightarrow\{0,1,2, \ldots, m\}
$$

satisfying

$$
\left\|\sigma_{j} g-g\right\|_{\infty} \leq 1
$$

for every $j=1,2, \ldots, m^{2}$ and

$$
\max _{y} \mathbf{P}[g(x)=y] \leq c / m,
$$

where $x$ is random-uniform in $\{a, b\}^{m^{2}}$.

Proof. Assume $a=0$ and $b=1$ for simplicity of notation. Let $k: \mathbb{N} \rightarrow$ $\{0,1,2, \ldots, m\}$ be the function satisfying $k(0)=0, k(j+1)=k(j)+1$ when $j \in \bigcup_{s=0}^{\infty}[2 s m, 2 s m+m-1]$ and $k(j+1)=k(j)-1$ for all other $j \in \mathbb{N}$. It is left to the reader to check that $g(x)=k\left(\|x\|_{1}\right)$ has the required properties. 
Fix some $v \in \mathbb{Z}^{d}$ with $|v|$ large and set $f=f(\omega):=\operatorname{dist}_{\omega}(0, v)$. Clearly, $f(\omega) \leq b|v|$ and, therefore, $|\gamma| \leq(b / a)|v|$, where $|\gamma|$ denotes the number of edges in $\gamma$. In particular, $f$ depends on only finitely many of the coordinates in $\omega$. Also, $|\gamma| \leq(b / a)|v|$ implies

$$
\sum_{e \in E} \mathbf{P}[e \in \gamma]=\mathbf{E}[|\gamma|] \leq(b / a)|v| .
$$

Fix $m:=\left\lfloor|v|^{1 / 4}\right\rfloor$ and let $S:=\{1, \ldots, d\} \times\left\{1, \ldots, m^{2}\right\}$. Let $c>0$ and $g=g_{m}$ be as in Lemma 3. Given $x=\left(x_{i, j}:\{i, j\} \in S\right) \in\{0,1\}^{S}$, let

$$
z=z(x):=\sum_{i=1}^{d} g\left(x_{i, 1}, \ldots, x_{i, m^{2}}\right) \mathbf{e}_{i},
$$

where $\left\{\mathbf{e}_{1}, \ldots, \mathbf{e}_{d}\right\}$ is the standard basis for $\mathbb{R}^{d}$. Define

$$
\tilde{f}(x, \omega):=\operatorname{dist}_{\omega}(z, v+z) .
$$

We think of $\tilde{f}$ as a function on the space $\tilde{\Omega}:=\{a, b\}^{S \cup E}$. Since $|z| \leq m d$, it follows that $|\tilde{f}-f| \leq 2 m d b$. In particular,

$$
\operatorname{var}(f) \leq \operatorname{var}(\tilde{f})+4 m d b \sqrt{\operatorname{var}(\tilde{f})}+4 m^{2} d^{2} b^{2} .
$$

It therefore suffices to find a good estimate for $\operatorname{var}(\tilde{f})$.

Let $e \in E$ be some edge. We want to estimate its influence:

$$
I_{e}(\tilde{f}):=\mathbf{P}\left[\sigma_{e} \tilde{f}(x, \omega) \neq \tilde{f}(x, \omega)\right]=2 \mathbf{P}\left[\sigma_{e} \tilde{f}(x, \omega)>\tilde{f}(x, \omega)\right] .
$$

Note that if the pair $(x, \omega) \in \tilde{\Omega}$ satisfies $\sigma_{e} \tilde{f}(x, \omega)>\tilde{f}(x, \omega)$, then $e$ must be on every $\omega$-geodesic from $z$ to $v+z$. Consequently, conditioning on $z$ and translating $\omega$ and $e$ by $-z$ gives

$$
I_{e}(\tilde{f})=2 \mathbf{P}\left[\sigma_{e} \tilde{f}(x, \omega)>\tilde{f}(x, \omega)\right] \leq 2 \mathbf{P}[e-z \in \gamma] .
$$

Let $Q$ be the set of edges $e^{\prime} \in E\left(\mathbb{Z}^{d}\right)$ such that $\mathbf{P}\left[e-z=e^{\prime}\right]>0$. The $L^{1}$ diameter of $Q$ is $O(m)$. [We allow the constants in the $O(\cdot)$ notation to depend on $d, a$ and $b$, but not on $v$.] Hence, the diameter of $Q$ in the $\operatorname{dist}_{\omega}$ metric is also $O(m)$ and, therefore, $|\gamma \cap Q| \leq O(m)$. However, the lemma gives

$$
\max _{z_{0}} \mathbf{P}\left[z=z_{0}\right] \leq(c / m)^{d} .
$$

By conditioning on $\gamma$ and summing over the edges in $\gamma \cap Q$, we therefore get

$$
\mathbf{P}[e \in \gamma+z \mid \gamma] \leq O(1) m^{1-d} .
$$

Consequently, (10) and the choice of $m$ give

$$
I_{e}(\tilde{f}) \leq O(1)|v|^{-1 / 4} .
$$


Also, (8) implies

$$
\sum_{e \in E} \mathbf{P}[e-z \in \gamma \mid z] \leq(b / a)|v| .
$$

Combining this with (10) therefore gives

$$
\sum_{e \in E} I_{e}(\tilde{f}) \leq 2(b / a)|v|
$$

Applying (11) yields

$$
\sum_{e \in E} \frac{I_{e}(\tilde{f})}{1+\left|\log I_{e}(\tilde{f})\right|} \leq O(1) \frac{|v|}{\log |v|} .
$$

On the other hand, $I_{S}(\tilde{f}) \leq b-a$ for $s \in S$. As $|S|=O(1)|v|^{1 / 2}$ and $\left\|\rho_{q} f\right\|_{\infty}=$ $O$ (1) for $q \in S \cup E$, we get from (12) and (2),

$$
\operatorname{var}(\tilde{f}) \leq O(1) \sum_{q \in E \cup S} \frac{I_{q}(\tilde{f})}{1+\left|\log I_{q}(\tilde{f})\right|} \leq O(1) \frac{|v|}{\log |v|}
$$

Therefore, Theorem 1 now follows from (9).

As alluded to in the Introduction, the proof would have been simpler if we could show that there is a $C>0$ such that $\mathbf{P}\left[\rho_{e} f(\omega) \neq 0\right]<|v|^{-C}$ holds with the possible exception of $|v| / \log |v|$ edges. It would be interesting to prove the closely related statement that the probability that $\gamma$ passes within distance 1 from $v / 2$ tends to zero as $|v| \rightarrow \infty$.

4. A proof of Talagrand's inequality (2). To prove (2), it clearly suffices to take $a=0$ and $b=1$. For $f:\{0,1\}^{J} \rightarrow \mathbb{R}$, consider the Fourier-Walsh expansion of $f$,

$$
f=\sum_{S \subset J} \hat{f}(S) u_{S}
$$

where $u_{S}(\omega)=(-1)^{S \cdot \omega}$ and $S \cdot \omega$ is shorthand for $\sum_{S \in S} \omega_{S}$. For each $p \in \mathbb{R}$ define the operator

$$
T_{p}(f):=\sum_{S \subset J} p^{|S|} \hat{f}(S) u_{S},
$$

which is of central importance in harmonic analysis. The Bonami-Beckner [2, 4] inequality asserts that

$$
\left\|T_{p} f\right\|_{2} \leq\|f\|_{1+p^{2}} .
$$

Set $f_{j}:=\rho_{j} f$. Because $\rho_{j} u_{S}=u_{S}$ if $j \in S$ and $\rho_{j} u_{S}=0$ if $j \notin S$, we have

$$
\hat{f}_{j}(S)= \begin{cases}\hat{f}(S), & j \in S, \\ 0, & j \notin S .\end{cases}
$$


Since $\|f\|_{2}^{2}=\sum_{S \subset J} \hat{f}(S)^{2}$, it follows that

$$
\operatorname{var}(f)=\sum_{\varnothing \neq S \subset J} \hat{f}(S)^{2}=\sum_{S \subset J} \sum_{j \in J} \hat{f}_{j}(S)^{2} /|S|=2 \sum_{j \in J} \int_{0}^{1}\left\|T_{p} f_{j}\right\|_{2}^{2} d p .
$$

Therefore, (13) gives

$$
\operatorname{var}(f) \leq 2 \sum_{j \in J} \int_{0}^{1}\left\|f_{j}\right\|_{1+p^{2}}^{2} d p .
$$

An instance of the Hölder inequality

$$
\mathbf{E}\left[\left|f_{j}\right|^{1+p^{2}}\right] \leq \mathbf{E}\left[f_{j}^{2}\right]^{p^{2}} \mathbf{E}\left[\left|f_{j}\right|\right]^{1-p^{2}}
$$

implies

$$
\begin{aligned}
\int_{0}^{1}\left\|f_{j}\right\|_{1+p^{2}}^{2} d p & \leq \int_{0}^{1}\left(\mathbf{E}\left[f_{j}^{2}\right]^{p^{2}} \mathbf{E}\left[\left|f_{j}\right|\right]^{1-p^{2}}\right)^{2 /\left(1+p^{2}\right)} d p \\
& =\left\|f_{j}\right\|_{2}^{2} \int_{0}^{1}\left(\left\|f_{j}\right\|_{1} /\left\|f_{j}\right\|_{2}\right)^{2\left(1-p^{2}\right) /\left(1+p^{2}\right)} d p \\
& \leq 2\left\|f_{j}\right\|_{2}^{2} \int_{1 / 2}^{1}\left(\left\|f_{j}\right\|_{1} /\left\|f_{j}\right\|_{2}\right)^{2\left(1-p^{2}\right) /\left(1+p^{2}\right)} d p .
\end{aligned}
$$

Let $s(p):=2\left(1-p^{2}\right) /\left(1+p^{2}\right)$. Since $s^{\prime}(p) \leq s^{\prime}(1)=-2$ when $p \in[1 / 2,1]$, the above gives

$$
\begin{aligned}
\int_{0}^{1}\left\|f_{j}\right\|_{1+p^{2}}^{2} d p & \leq 2\left\|f_{j}\right\|_{2}^{2} \int_{s(1 / 2)}^{s(1)}\left(\left\|f_{j}\right\|_{1} /\left\|f_{j}\right\|_{2}\right)^{s} \frac{d s}{s^{\prime}(p)} \\
& \leq\left\|f_{j}\right\|_{2}^{2} \int_{0}^{6 / 5}\left(\left\|f_{j}\right\|_{1} /\left\|f_{j}\right\|_{2}\right)^{s} d s \\
& =\left\|f_{j}\right\|_{2} \frac{1-\left(\left\|f_{j}\right\|_{1} /\left\|f_{j}\right\|_{2}\right)^{6 / 5}}{\log \left(\left\|f_{j}\right\|_{2} /\left\|f_{j}\right\|_{1}\right)} .
\end{aligned}
$$

Now (14) implies

$$
\operatorname{var}(f) \leq 2 \sum_{j \in J}\left\|f_{j}\right\|_{2}^{2} \frac{1-\left(\left\|f_{j}\right\|_{1} /\left\|f_{j}\right\|_{2}\right)^{6 / 5}}{\log \left(\left\|f_{j}\right\|_{2} /\left\|f_{j}\right\|_{1}\right)},
$$

from which (2) follows.

REMARK. It is worth noting that the tail estimate (1) can be derived from the variance inequality (2). Indeed, let $f=\operatorname{dist}_{\omega}(0, v)$ and $u \in(0,3 / 4)$. Set $s=s(u):=\inf \{t \geq 0: \mathbf{P}[f(\omega)>t]<u\}$, and define $g(\omega):=\max \{f(\omega), s\}$. It follows from (2) that

$$
\operatorname{var}(g) \leq C u|v| /\left(1+\min _{e} \log \left(\left\|\rho_{e} g\right\|_{2} /\left\|\rho_{e} g\right\|_{1}\right)\right)
$$


and, hence, $\operatorname{var}(g) \leq C u|v| / \log (1 / u)$. Therefore, there is a constant $C^{\prime}$ such that

$$
\mathbf{P}\left[f(x)>s(u)+C^{\prime} \sqrt{|v| / \log (1 / u)}\right]<u / 2 .
$$

That is, $s(u / 2) \leq s(u)+C^{\prime} \sqrt{|v| / \log (1 / u)}$. Induction gives for $n=1,2, \ldots$,

$$
s\left(2^{-n}\right) \leq s(1 / 2)+O(1) \sqrt{n|v|},
$$

which is the "upper tail" bound from (1). The lower tail is treated similarly. (This proof is fairly general. Using the more specific arguments from Section 3, a slight improvement for the tail estimates in certain ranges may be obtained.)

Acknowledgment. We are most grateful to Elchanan Mossel for very useful discussions.

\section{REFERENCES}

[1] Baik, J., Deift, P. and Johansson, K. (1999). On the distribution of the length of the longest increasing subsequence of random permutations. J. Amer. Math. Soc. 12 1119-1178.

[2] BeCKner, W. (1975). Inequalities in Fourier analysis. Ann. of Math. 102 159-182.

[3] Boвкov, S. G. and Houdre, C. (1999). A converse Gaussian Poincare-type inequality for convex functions. Statist. Probab. Lett. 44 281-290.

[4] Bonami, A. (1970). Etude des coefficients Fourier des fonctiones de $L^{p}(G)$. Ann. Inst. Fourier (Grenoble) 20 335-402.

[5] Bourgain, J., Kahn, J., Kalai, G., Katznelson, Y. and Linial, N. (1992). The influence of variables in product spaces. Israel J. Math. 77 55-64.

[6] Deuschel, J.-D. and Zeitouni, O. (1999). On increasing subsequences of I.I.D. samples. Combin. Probab. Comput. 8 247-263.

[7] Durrett, R. (1999). Perplexing problems in probability. Progr. Probab. 44 1-33.

[8] FRIEdGUt, E. (200X). Influences in product spaces, KKL and BKKKL revisited. Preprint.

[9] Johansson, K. (2000). Transversal fluctuations for increasing subsequences on the plane. Probab. Theory Related Fields 116 445-456.

[10] Kahn, J., KAlai, G. and Linial, N. (1988). The influence of variables on Boolean functions. In Proceedings of the 29th Annual Symposium on Foundations of Computer Science 68-80. IEEE Computer Science Press, Washington, DC.

[11] Kesten, H. (1986). Aspects of first passage percolation. École d'Été de Probabilités de SaintFlour, XIV. Lecture Notes in Math. 1180 125-264. Springer, Berlin.

[12] Kesten, H. (1993). On the speed of convergence in first passage percolation. Ann. Appl. Probab. 3 296-338.

[13] Ledoux, M. (2001). The Concentration of Measure Phenomenon. Amer. Math. Soc., Alexandria, VA.

[14] Newman, C. M. and PiZA, M. (1995). Divergence of shape fluctuations in two dimensions. Ann. Probab. 23 977-1005.

[15] Pemantle, R. and Peres, Y. (1994). Planar first-passage percolation times are not tight. In Probability and Phase Transition (G. Grimmett, ed.) 261-264. Kluwer, Dordrecht. 
[16] Talagrand, M. (1994). On Russo's approximate zero-one law. Ann. Probab. 22 1576-1587.

[17] TALAgRAnD, M. (1995). Concentration of measure and isoperimetric inequalities in product spaces. Inst. Hautes Études Sci. Publ. Math. 81 73-205.

I. BENJAMINI

WEIZMANn Institute of SCIENCE

REHOVOT 76100

ISRAEL

E-MAIL: itai@wisdom.weizmann.ac.il

URL: www.wisdom.weizmann.ac.il/ itai/
G. KALAI

HEBREW UNIVERSITY

GIVAT RAM

JERUS ALEM 91904

ISRAEL

E-MAIL:kalai@math.huji.ac.il

URL: www.ma.huji.ac.il/ kalai/

O. SCHRAMM

MICROSOFT RESEARCH

ONE MICROSOFT WAY

REDMOND, WASHINGTON 98052

E-MAIL: schramm@microsoft.com

URL: research.microsoft.com/ schramm/ 